This item was submitted to Loughborough's Institutional Repository (https://dspace.lboro.ac.uk/) by the author and is made available under the following Creative Commons Licence conditions.

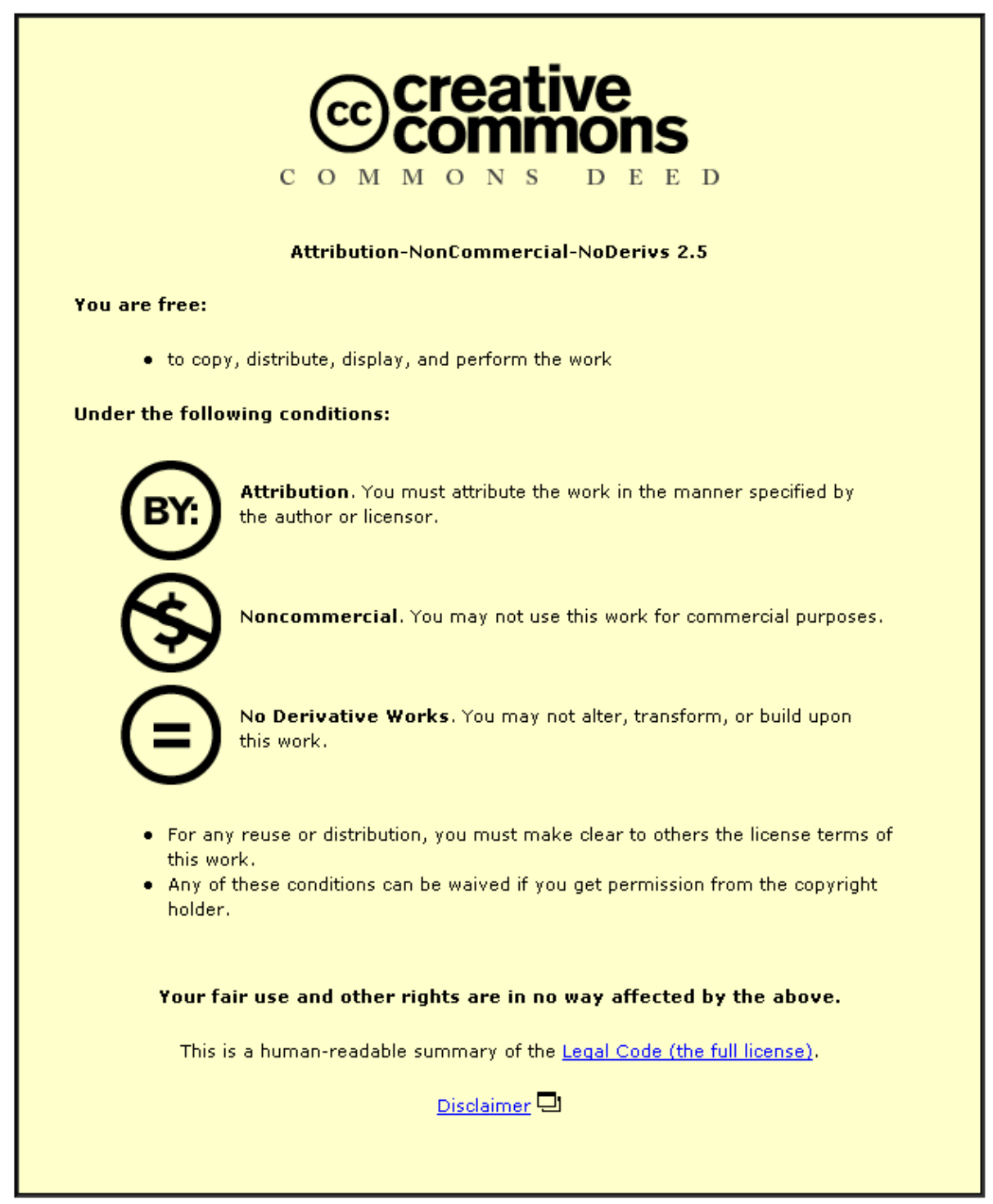

For the full text of this licence, please go to: http://creativecommons.org/licenses/by-nc-nd/2.5/ 


\title{
X-ray-based machine vision system for distal locking of intramedullary nails
}

\author{
F Junejo ${ }^{1}$, K Bouazza-Marouf ${ }^{1 *}$, D Kerr ${ }^{1}$, A J Taylor ${ }^{1}$, and G J S Taylor ${ }^{2}$ \\ ${ }^{1}$ Wolfson School of Mechanical and Manufacturing Engineering, University of Loughborough, Loughborough, \\ Leicestershire, UK \\ ${ }^{2}$ Department of Orthopaedic Surgery, Glenfield Hospital, Leicester, UK
}

The manuscript was received on 14 March 2006 and was accepted after revision for publication on 26 January 2007.

DOI: 10.1243/09544119JEIM166

\begin{abstract}
In surgical procedures for femoral shaft fracture treatment, current techniques for locking the distal end of intramedullary nails, using two screws, rely heavily on the use of two-dimensional X-ray images to guide three-dimensional bone drilling processes. Therefore, a large number of X-ray images are required, as the surgeon uses his/her skills and experience to locate the distal hole axes on the intramedullary nail. The long-term effects of X-ray radiation and their relation to different types of cancer still remain uncertain. Therefore, there is a need to develop a surgical technique that can limit the use of X-rays during the distal locking procedure. A robotic-assisted orthopaedic surgery system has been developed at Loughborough University to assist orthopaedic surgeons by reducing the irradiation involved in such operations. The system simplifies the current approach as it uses only two near-orthogonal X-ray images to determine the drilling trajectory of the distal locking holes, thereby considerably reducing irradiation to both the surgeon and patient. Furthermore, the system uses robust machine vision features to reduce the surgeon's interaction with the system, thus reducing the overall operating time. Laboratory test results have shown that the proposed system is very robust in the presence of variable noise and contrast in the X-ray images.
\end{abstract}

Keywords: robotic/computer-assisted orthopaedic surgery, intramedullary nailing, distal locking

\section{INTRODUCTION}

The femur, or thigh bone, is the longest, largest, and strongest bone of the human body [1]. Femoral shaft fractures commonly occur as a result of road accidents, falls, and gunshot wounds. In addition, osteoporosis in elderly people dramatically increases the risk of femur fracture. Closed intramedullary nailing (IMN) has shown its efficacy in the treatment of diaphyseal fractures of long bones, especially of complex fractures of the femur. The concept behind IMN is to insert a nail (a stainless steel tube) into the bone canal from the proximal end. This allows

\footnotetext{
* Corresponding author: Wolfson School of Mechanical and Manufacturing Engineering, University of Loughborough, Loughborough, Leicestershire LE11 3TU, UK. email: K.Bouazzamarouf@lboro.ac.uk
}

the surgeon to perform both the reduction and stabilization procedures without the complications of opening the fracture site, thereby reducing further damage to the traumatized area. This results in lower blood loss, lower infection risk, shorter hospitalization time, and reduced morbidity. Owing to these advantages, IMN has become the standard treatment for long bone fractures.

Although conventional IMN with only proximal locking may provide adequate stabilization of midshaft fractures, the concept of distal locking was introduced in order to provide more effective fixation. Distal locking involves attaching the nail to the bone fragment at the distal end of the femur by means of screws, as shown in Fig. 1, to provide rotational and axial stability.

A number of problems are faced by orthopaedic surgeons during distal locking of intramedullary nails, the most serious being as follows. 


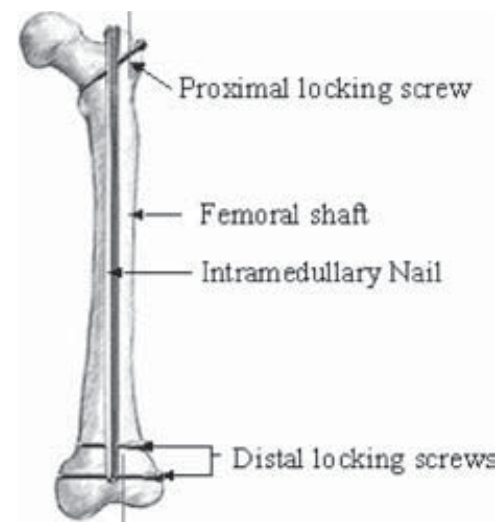

Fig. 1 Femoral shaft fracture treatment using intramedullary nailing

1. The intramedullary nail may deform by several millimetres on its passage through the medullary canal to conform to the bone canal shape [2]. Therefore, without compensation for nail deformation, it is impossible to place the distal locking screws correctly using an external guide attached to the proximal end of the nail, as is used for proximal locking screws.

2. The use of two-dimensional X-ray images to guide three-dimensional bone drilling procedures means that a large number of X-ray images are required. The surgeon has mentally to correlate the surgical tool and desired drilling trajectory without direct visual feedback. Hence, sets of $\mathrm{X}$-ray images must be frequently acquired to ascertain each new position of the surgical tool.

These difficulties make the insertion of the distal locking screws the most demanding step of the IMN operation [2]. In order to overcome these difficulties, many devices have been developed to assist orthopaedic surgeons in performing distal locking. Examples include proximally mounted targeting devices [3], image intensifier mounted devices [4], laser devices [5], stereo fluoroscopy [6], and magnetic [7] and mechanical guides [8]. However, all of these devices and techniques have deficiencies. For example, they lack versatility (i.e. are only selectively applicable), are unstable and not easy to use, are time consuming, or are not sufficiently accurate.

Free-hand techniques are currently used by most surgeons as the best available method for the distal locking procedure. However, surgeons rely heavily on hand-eye coordination during the entire procedure, and therefore both the duration and outcome of the procedure are highly dependent upon the skill and the experience of the surgeon. Madan and Blakeway [9] have demonstrated that the average time of radiation exposure during femoral nailing reduces to one-third when performed by a consultant rather than a middle-grade surgeon. The free-hand technique uses the so-called 'perfect circle approach' in which the position of the C-arm fluoroscopy unit in relation to the stationary leg is adjusted using $\mathrm{X}$-ray imaging until the two distal holes appear as near perfect circles rather than as ellipses. The surgeon then uses additional X-ray images to adjust the entry point and orientation of the drill bit to align it with the corresponding distal hole axis. Drilling proceeds incrementally, with each advance verified with a new pair of near-orthogonal X-ray images, resulting in excessive irradiation to the surgeon and patient. According to Mehlman and DiPasquale [10] the total radiation exposure time for femoral intramedullary nailing with proximal and distal locking varies between 3.1 and $31.4 \mathrm{~min}$, with distal locking alone occupying up to 30 to 50 per cent of the total radiation exposure time. Therefore, there is concern among orthopaedic surgeons about the high levels of exposure to radiation associated with intraoperative $\mathrm{C}$-arm fluoroscopy during the distal locking procedure [11], as there is evidence that carcinogenic potential exists from low-dose, low-energy radiation [12].

The risk of intraoperative radiation to orthopaedic surgeons has recently been the impetus for many researchers to develop surgical techniques that would limit the need for fluoroscopy during distal locking of intramedullary nails. Nolte and co-workers $[\mathbf{1 3}, \mathbf{1 4}]$ developed a computer-assisted free-hand navigation system that uses intraoperative $\mathrm{X}$-ray images as a basis for real-time navigation of surgical tools. It separates image acquisition from the surgical procedure, as it offers continuous intraoperative guidance to surgeons by producing real-time positioning of the surgical tool overlaid on stored X-ray images. The system consists of an optical tracking system, that is, an optically tracked calibration grid attached to the image intensifier of the C-arm and optically tracked markers placed on both the implant and the surgical tool. There are other commercially available surgical navigation systems based on computerassisted fluoroscopy, such as SurgiGATE (Medivision, Switzerland) and Fluoronav (Medtronic, USA). Phillips and co-workers $[\mathbf{1 5}, 16]$ have developed a computerassisted orthopaedic surgery system which also uses optical tracking cameras and includes a lockable passive arm. The arm is used to align a drill guide with the computed trajectory using a graphical display. However, although these surgical assist systems reduce the irradiation to the surgeon, they may result in a longer overall procedure time in comparison with the 
free-hand technique [17]. Furthermore, these systems require an unobstructed line of sight between the optical tracking cameras and infrared LEDs mounted on the image intensifier and the surgical tool (or drill guide), resulting in a limitation of free movement by the surgical personnel.

The system developed at Loughborough University by Bouazza-Marouf and co-workers [18-21] for robotic-assisted internal fixation of hip fractures and distal locking of intramedullary nails uses a calibration frame, shown in Fig. 2, and a C-arm X-ray unit. The calibration frame contains two lateral and two anterior-posterior (AP) calibration plates, with each plate containing an array of embedded fiducial markers (stainless steel balls). The drilling trajectory is computed from two near-orthogonal intraoperative $\mathrm{X}$-ray images taken with the calibration frame in position around the body part of interest. The radiopaque reference markers (inserted in the radiolucent plates of the calibration frame) which are visible on each X-ray view and image points of interest (e.g. as shown in Fig. 3) are used to establish the drilling trajectory with respect to the calibration frame coordinate system. Speed, robustness, reliability, and repeatability are of paramount importance when introducing any new technology into the operating theatre. Therefore, the novelty of the work presented

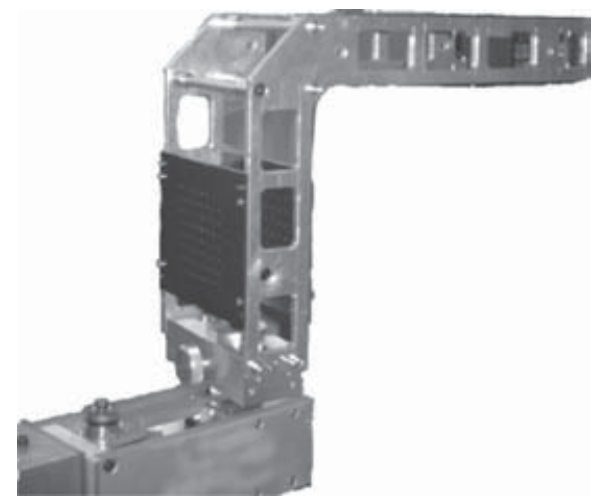

Fig. 2 Calibration frame in this paper lies in the robustness of the machine vision system and the off-line CAD modelling of the intramedullary nail. Look-up tables are obtained by off-line CAD modelling of nails of different shapes and dimensions, and all the steps involved during image analysis have been fully automated to make the vision system user independent and very efficient in terms of time taken to determine the drilling trajectory.

Furthermore, the adopted machine vision system is shown in section 3 to be very robust in the presence of variable noise and contrast in the $\mathrm{X}$-ray images and in terms of variable magnification. Therefore, the benefits of this approach are as follows.

1. The use of off-line modelling of the nail reduces the length of time of the surgical procedure.

2. The method allows modelling of any shape of intramedullary nail accurately; therefore, nails from different manufacturers can be modelled.

3. User-independent image analysis.

4. Robustness of the machine vision system.

It should be noted that there are two types of intramedullary nail - slotted and solid. Slotted nails may have large deformation owing to the relative low stiffness compared with solid nails. The latter nails have virtually negligible local (at the distal holes) deformation to affect the circularity of the holes. This work relates to solid intramedullary nails.

\section{LOUGHBOROUGH ORTHOPAEDIC ASSISTANT SYSTEM: X-RAY IMAGE ANALYSIS}

The Loughborough robotic-assisted orthopaedic surgery system described in section 1 uses a calibration frame for calibration and registration purposes rather than relying on an optical tracking system such as Optotrak. Two near-orthogonal lateral and AP X-ray images of the distal end of the intramedullary nail are taken with the calibration frame
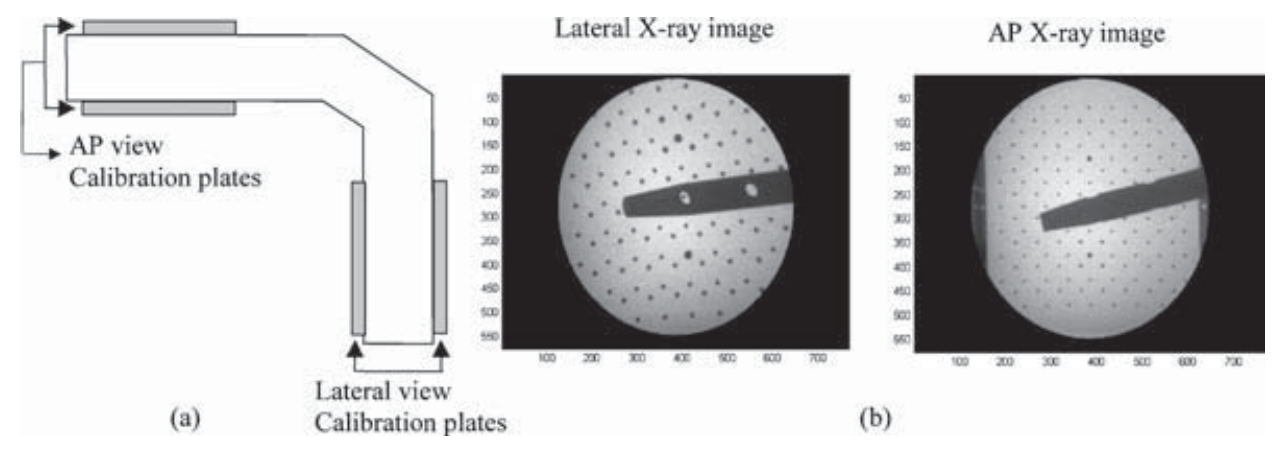

Fig. 3 (a) Calibration frame and (b) typical lateral and AP X-ray images 


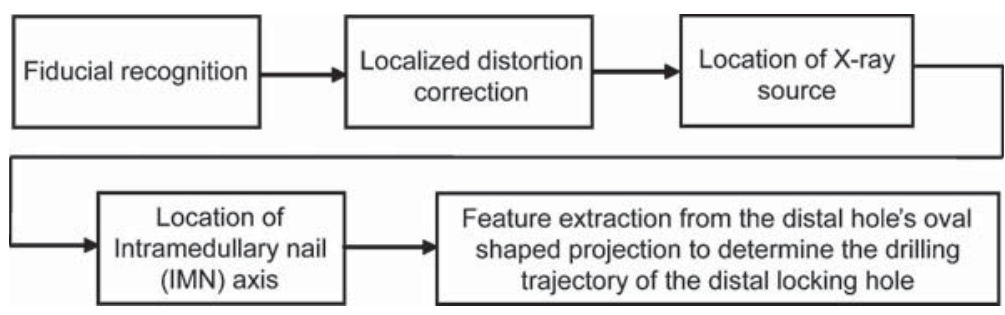

Fig. 4 Flow diagram of X-ray image analysis

in position. The calibration frame and typical X-ray images are shown in Fig. 3.

Simulation of the distal locking procedure was performed using a specially designed test rig incorporating a cylindrical nail of $14 \mathrm{~mm}$ diameter containing two distal holes of $7 \mathrm{~mm}$ diameter spaced $30 \mathrm{~mm}$ apart to represent a typical intramedullary nail. The nail can be rotated accurately by known angles so that results obtained from image analysis can then be verified. The experimental results are given in section 3. For clarity, the remainder of this section describes the machine vision system together with the enhancements made in order to reduce the length of time of the surgical procedure in determining the drilling trajectory for IMN distal locking. The image analysis protocol depicted by the flow diagram of Fig. 4 is followed once the lateral and AP intraoperative X-ray images are acquired.

\subsection{Fiducial recognition}

$\mathrm{X}$-ray photogrammetry techniques work by introducing fiducials into radiographic images to reconstruct three-dimensional locations of known object points. Therefore, firstly, automatic fiducial recognition is carried out to determine the image (pixel) coordinates of all the visible fiducials, in both lateral and AP X-ray images. For fiducial recognition, this study employs a localized thresholding approach by dividing the whole image into small subregions in which each fiducial is processed individually. Any error in fiducial recognition can induce errors in distortion correction and focal point determination. Therefore, any partially imaged fiducials on the edge of the X-ray image and fiducials touching the edges of the nail (shown in Fig. 5) are automatically identified and are not considered in the subsequent analysis.

\subsection{Localized distortion correction}

$\mathrm{X}$-ray images have inherent distortion, mainly owing to the internal geometry of the image intensifier and the influence of magnetic fields on the electron acceleration within the X-ray tube. Therefore, localized distortion correction based on fiducial neighbourhood

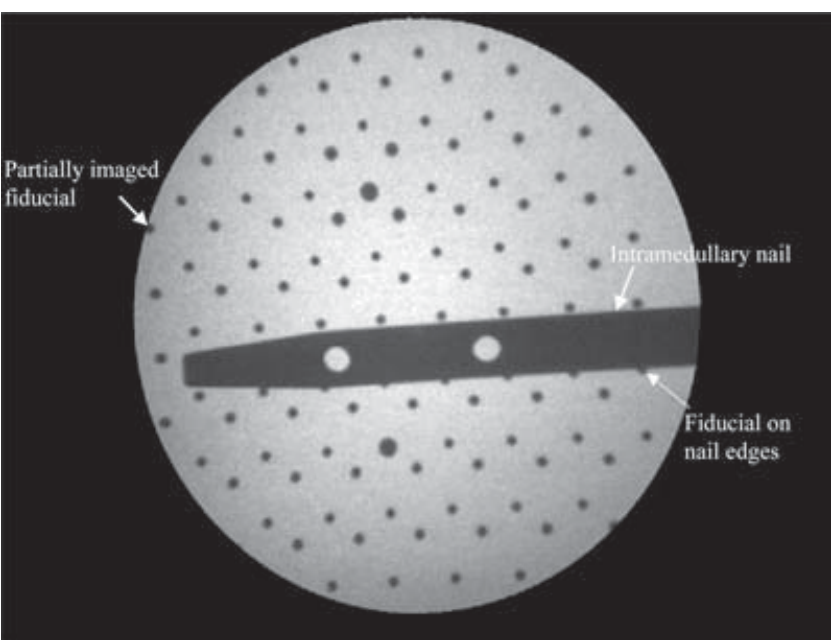

Fig. 5 Fiducial recognition in a lateral X-ray image

scanning has been undertaken for intraoperative distortion correction of the X-ray images $[\mathbf{1 9}, \mathbf{2 1}]$. This approach allows data obtained directly from actual intraoperative images to be used to perform distortion correction. As a result, assumptions do not have to be made with regard to magnetic influence in the operating room, the internal geometry of the image intensifier tube, or the orientation of the C-arm unit.

\subsection{Location of the X-ray source}

$\mathrm{X}$-rays can be considered to be a special case of central projection, in which all the rays pass through a perspective centre, or focal point $[\mathbf{1 9}, \mathbf{2 1}]$. This point is not identifiable as a physical feature of the X-ray machine but lies at a virtual point behind the X-ray tube. Therefore, in order to convert two-dimensional image information into a three-dimensional position, it is essential to determine the focal point in both lateral and AP views. Once fiducial recognition has been performed, both the image (pixel) and world $(\mathrm{mm})$ coordinates of fiducials appearing within the field of view are known. Focal lines are then constructed to determine the location of the X-ray source. Construction of the focal lines is accomplished by selecting image points within the intraoperative 
$\mathrm{X}$-ray image and assigning their corresponding world coordinates to a point on each of the two calibration plates in the relevant view. For this purpose, centres of some of the detected fiducials on the XRII calibration plate (i.e. the calibration plate nearest to the X-ray image intensifier) are selected as image points, as their world coordinates are already known, thereby avoiding any calculation error. For each of these image points a corresponding point on the adjacent source calibration plate (i.e. the calibration plate nearest to the X-ray source) is obtained, as described in reference $[\mathbf{2 1}]$. The intersection of each pair of focal lines is obtained and, for pairs that do not intersect owing to the nature of X-ray imaging systems, the point of closest approach is determined as the intersection. The centre of gravity of the volume of this set of intersection points is then used as the focal point, i.e. the estimated location of the $\mathrm{X}$-ray source.

\subsection{Location of the nail axis}

In order to determine the nail axis in space, automatic nail border detection is carried out in both lateral and AP views. The borderlines of the nail can be obtained by analysing the two major transitions in grey level values within a profile drawn across the nail. However, measuring the nail edges only once can lead to potential errors, such as the presence of a fiducial within the selected profile. Therefore, for nail edge detection, multiple parallel line profiles on both sides of the distal hole projection are generated, as shown in Fig. 6.

By assuming that the nail axis coincides with the centre of the nail borderlines, the two boundary edges for the nail are bisected to determine the nail axis in both the lateral and AP views. In the lateral view it is possible to find two points on the centre-

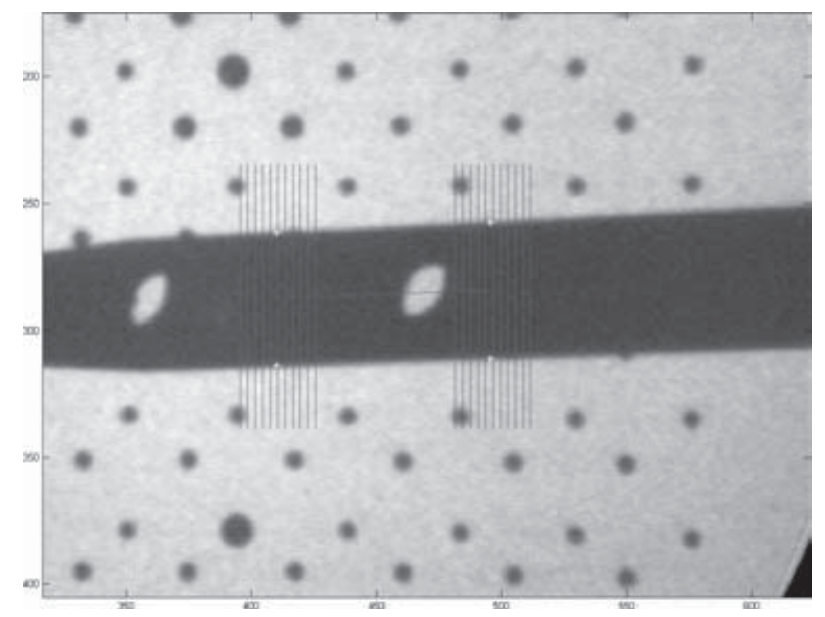

Fig. 6 Nail edge detection in lateral view line of the nail. Once these points are known in the image plane, the triangulation technique described in reference [21] is used to find their corresponding world coordinates on the calibration plate. Both these points in conjunction with the lateral view focal point define a plane. A similar approach is carried out for the AP view. Once these planes are defined in the lateral and $\mathrm{AP}$ views, their intersection defines the nail axis in space $[\mathbf{1 6}, \mathbf{2 0}]$, as shown in Fig. 7.

\subsection{Determination of the drilling axis for distal holes}

The features that are used to determine the drilling trajectory are the angle $\lambda$ (shown in Fig. 8) between the major axis of the oval and the nail axis, the target point (which is the intersection of the distal hole axis and the nail axis), and the angle $\phi$ (shown in Fig. 10) between the X-ray imaging axis and the nail axis. These features are described below.

The angle of the major axis of the oval-shaped distal hole projection changes significantly with respect to the nail axis when the nail is rotated about its own axis. Therefore, the region containing the distal hole projection is automatically selected from the lateral view $\mathrm{X}$-ray image, and image segmentation is performed to determine the major axis of the oval. The angle $\lambda$ between the major axis of the oval and the nail axis is then determined as a characteristic measurement.

On occasions, however, the image of the hole will contain a fiducial at the edge or entirely within the oval. Therefore, once the region containing the distal locking hole is selected, a check is made in the surroundings of the oval to determine the presence of any fiducial. In the case of a fiducial lying completely within the oval, it is relatively easy to convert that fiducial into part of the oval. However, in the case of a fiducial lying on the edge of the oval, e.g. as shown in Fig. 9, an algorithm has been developed to remove the fiducial region within the oval.

The desired drilling trajectory must pass through the centre of the distal hole, i.e. the target point, which is the intersection of the nail axis with the distal hole axis. This point is found by intersecting the nail axis with the line of sight that passes through the centre of the distal hole. In order to construct this line of sight, the image (pixel) coordinates of the centre of the oval-shaped distal hole projection are determined using the lateral X-ray image. Then, application of the triangulation technique to this measured pixel location allows the corresponding calibration plate intersection point, $\mathrm{P}_{\text {Source, }}$ to be calculated. Therefore, a line of sight is defined using 


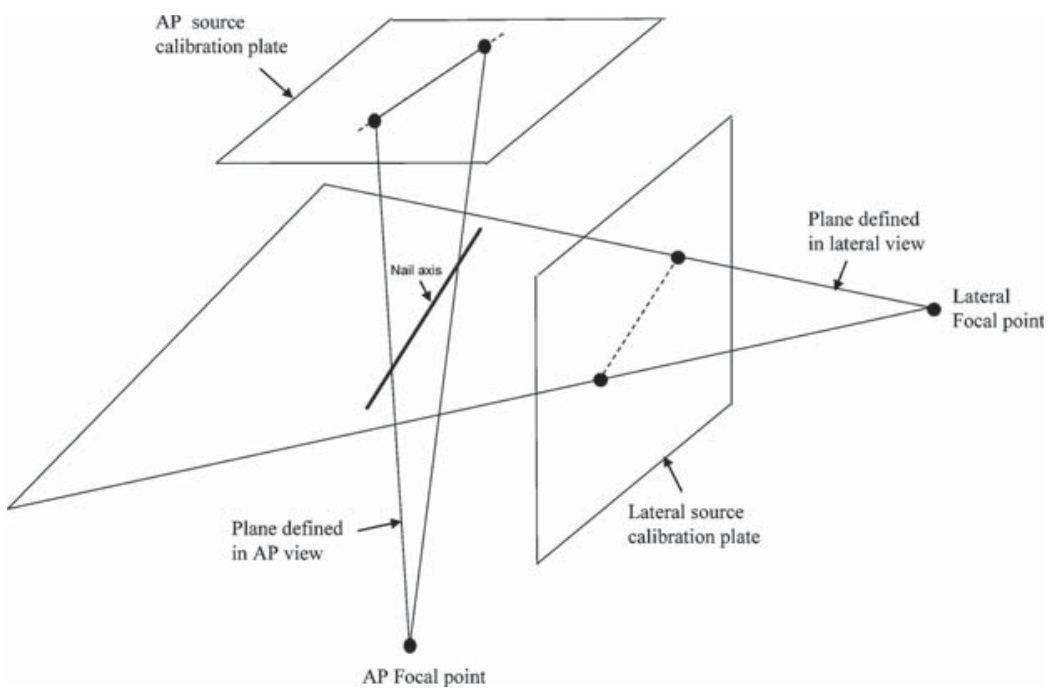

Fig. 7 Recovery of the nail axis in space

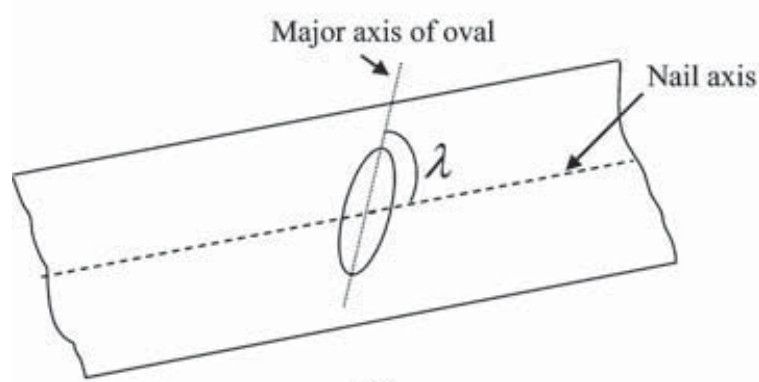

(a)

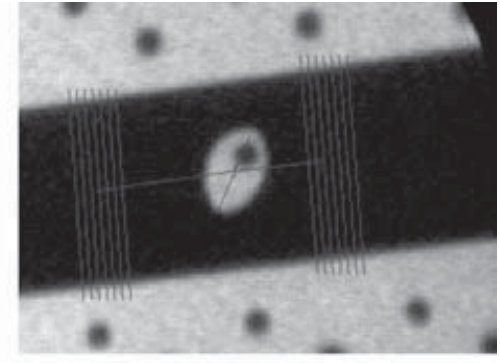

(b)

Fig. 8 Angle between the major axis of the oval and the nail axis: (a) concept; (b) implementation using image analysis

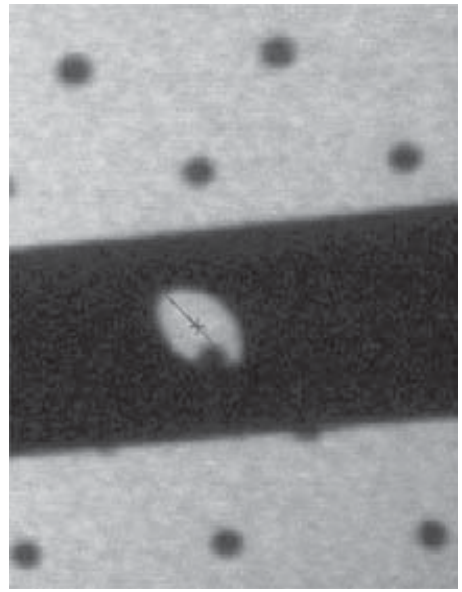

Fig. 9 Major axis determination of the oval when a fiducial lies at the edge of the oval

the known focal point of the lateral view in conjunction with $\mathrm{P}_{\text {Source }}$ which passes through the target point $\mathrm{O}$. The angle $\phi$ between the nail axis and line of sight (defined by the line passing through the target point $\mathrm{O}$ and lateral focal point), as shown in Fig. 10, yields the orientation of the nail with respect to the X-ray imaging axis.

With the adopted technique, in addition to the angles $\lambda$ and $\phi$ and the coordinates of the centre of the distal hole (target point), it is also necessary to determine the rotation of the nail, $\theta$, about its long axis with respect to the line of sight, as shown in Fig. 11. For this purpose, three-dimensional modelling of the nail is done using the manufacturer's data, and then, using this model, simulation for a range of values of $\theta$ and $\phi$ is performed. For each unique rotation of the nail, the oval-shaped projection of the distal locking hole is automatically analysed to determine the angle between the major axis of the oval and the nail axis, i.e. $\lambda$. This value of $\lambda$, obtained from the modelling of the nail, together with the corresponding values of $\theta$ and $\phi$, is then imported into Matlab to build a look-up table. The angles $\lambda$ and $\phi$ obtained from the image analysis are then entered as input into the look-up-table software to obtain the rotation angle $\theta$. The drilling trajectory is 


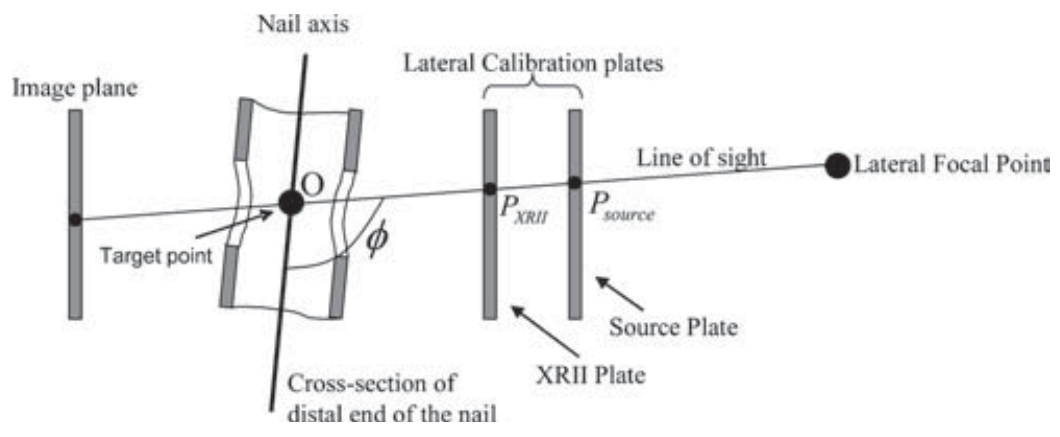

Fig. 10 Determination of the centre of the distal hole and $\phi$

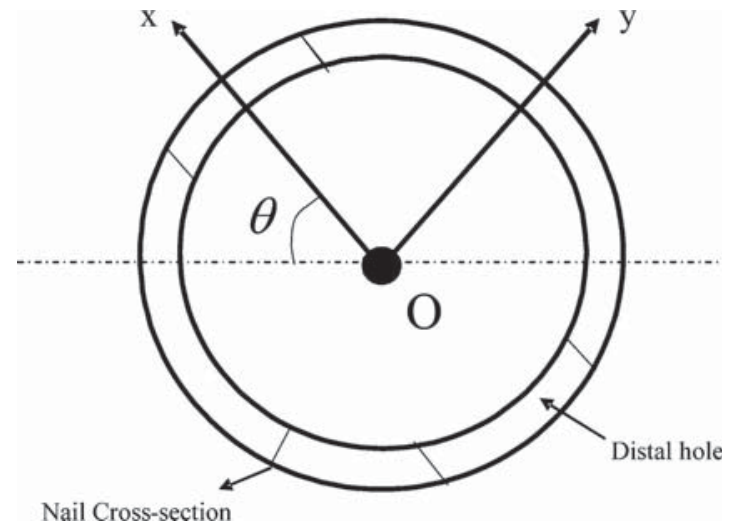

Fig. 11 Rotation of the nail about its long axis

then defined using the derived position of the centre of the distal hole (target point) and the angles $\theta$ and $\phi$.

\section{ACCURACY EVALUATION OF THE MACHINE VISION SYSTEM}

In order to evaluate the accuracy of the computer vision system, the drilling trajectory, as computed with respect to the calibration frame, was compared with the true drilling trajectory obtained using theodolite measurements. The following procedure was adopted.

1. Several points on the surface of the lateral XRII calibration plate were measured using two theodolites to define the calibration frame coordinate system with respect to the theodolite frame of reference, i.e. to obtain the homogeneous transform ${ }_{\mathrm{TH}}^{\mathrm{CF}} \mathbf{T}$, where $\mathrm{CF}$ and $\mathrm{TH}$ refer to calibration frame and theodolite respectively.

2. A cylindrical nail of $14 \mathrm{~mm}$ diameter containing two distal holes of $7 \mathrm{~mm}$ was attached to a test rig. To cover a wide range of possible nail orientations likely to be encountered during the surgical procedure, this test rig was used to simulate different rotations, $\theta$, of the nail about its own axis, with the nail at different orientations, $\phi$, with respect to the X-ray imaging axis. In order to define the true trajectory of the distal locking hole, a testpiece comprising a solid cylinder of $7_{-0.01}^{+0.00} \mathrm{~mm}$ diameter with an integral concentric disc of $50 \mathrm{~mm}$ diameter at one end was fitted in the hole. On the surface of this disc, five points, including the centre, were measured with respect to the theodolite frame of reference to determine the central axis ${ }^{\mathrm{TH}} v$ of the testpiece and thus the distal hole axis with respect to the theodolite frame of reference.

3. The central axis of the distal locking hole with respect to the calibration frame, obtained as

$$
{ }^{\mathrm{CF}} v={ }_{\mathrm{TH}}^{\mathrm{CF}} \mathbf{T} \cdot{ }^{\mathrm{TH}} v={ }_{\mathrm{CF}}^{\mathrm{TH}} \mathbf{T}^{-1} \cdot{ }^{\mathrm{TH}} v
$$

was then compared with the computed drilling trajectory described in section 2 .

Additionally, the position of the lateral X-ray source was varied, as discussed in section 3.1, to evaluate the influence of X-ray magnification on the accuracy of the vision system.

The results from the laboratory trials are summarized in Fig. 12. The corresponding numbers in Figs 12(a) and (b) represent the entry and exit points respectively for the nine drilling trajectories. Analysis has shown that a mean positional error of $0.81 \mathrm{~mm}$ (the worst case being $1.18 \mathrm{~mm}$, as depicted by trajectory 5) and a mean angular error of $1.01^{\circ}$ (the worst case being $1.25^{\circ}$, as depicted by trajectory 2) were obtained. These values are considered acceptable for inserting a guide wire of $2.5 \mathrm{~mm}$ diameter into the distal locking hole of $7 \mathrm{~mm}$ diameter (or a $5 \mathrm{~mm}$ diameter distal locking hole) and the subsequent screw insertion.

In light of previous research by Phillips and co-workers $[15,16]$, and in consultation with orthopaedic surgeons, successful drilling of the pilot hole (for the locking screw) using a guide wire will 


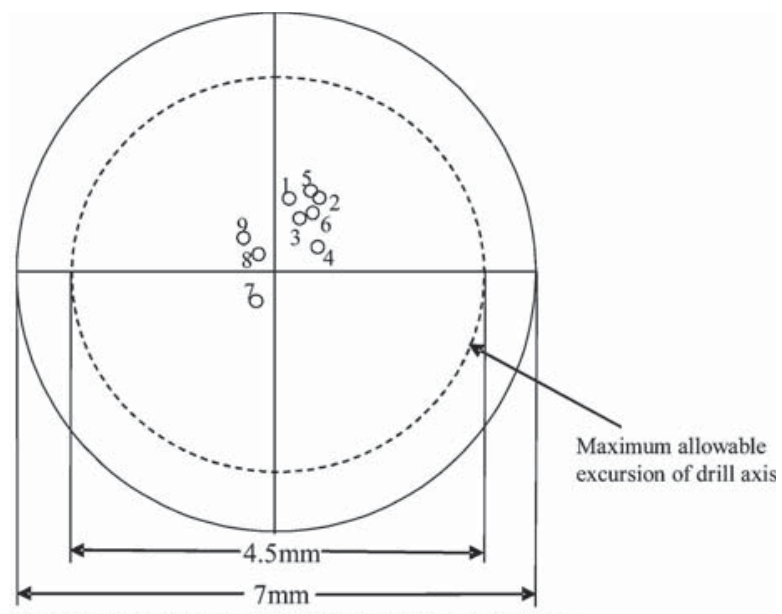

(a) Entry point for drilling trajectory at the distal locking hole

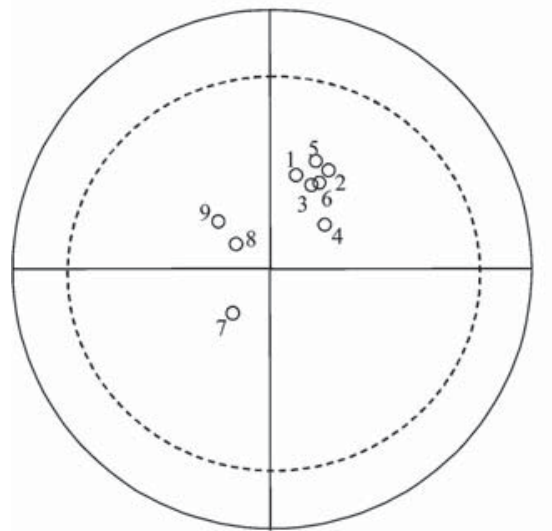

(b) Exit point for drilling trajectory at the distal locking hole

Fig. 12 Results from accuracy evaluation of the computer vision system

result in the successful locking of distal holes of an intramedullary nail. Therefore, based on the results obtained from the laboratory trials presented in this paper, the proposed system provides the accuracy required for a successful insertion of distal locking screws.

To evaluate the robustness of the system, the effects of the position of the C-arm X-ray unit with respect to the calibration frame and of contrast and noise variations in the X-ray images have been studied, as discussed below.

\subsection{Effect of X-ray source position on the lateral view image}

In the adopted procedure, the $\mathrm{C}$-arm image intensifier is positioned as close as possible to the calibration frame plates in the AP view, but in the lateral view the position of the image intensifier (and thus the position of the X-ray source) can vary. Therefore, the effect of positioning of the X-ray source has been evaluated.
In X-ray imaging, object points nearer to the X-ray source experience higher magnification than object points nearer to the image plane. Therefore, during laboratory trials, in order to determine the robustness of the image analysis in predicting nail rotation about its long axis at different magnifications, three different positions of the X-ray source with respect to the calibration frame were used for each set of rotations of $(\varphi$ and $\theta)$ of the nail. During the trials, the three values of $D$ (shown in Fig. 13) used were 345 , 395, and $445 \mathrm{~mm}$, i.e. a difference of $50 \mathrm{~mm}$ between the positions. The latter distance represents a standard position of the X-ray source for the lateral image.

Analysis showed that, for different values of $D$, the maximum deviation in predicting the rotation of the nail about its long axis is found to be $0.08^{\circ}$. This shows the robustness of the image segmentation techniques used and is an indication of its suitability for clinical application.

\subsection{Influence of contrast and noise variations on image analysis}

During this study, special attention has been given to the effect of variable contrast and noise in the $\mathrm{X}$-ray images on the image analysis. To measure accurately the performance of the vision system, the combined effects of noise and contrast must be evaluated. Since the contrast of an X-ray image is controlled by a combination of X-ray tube voltage and current, different combinations of voltage and current were used during laboratory trials to achieve a wide range of contrast in the images. Noise can prove to be a major limiting factor in image segmentation, as image quality degrades very quickly when noise

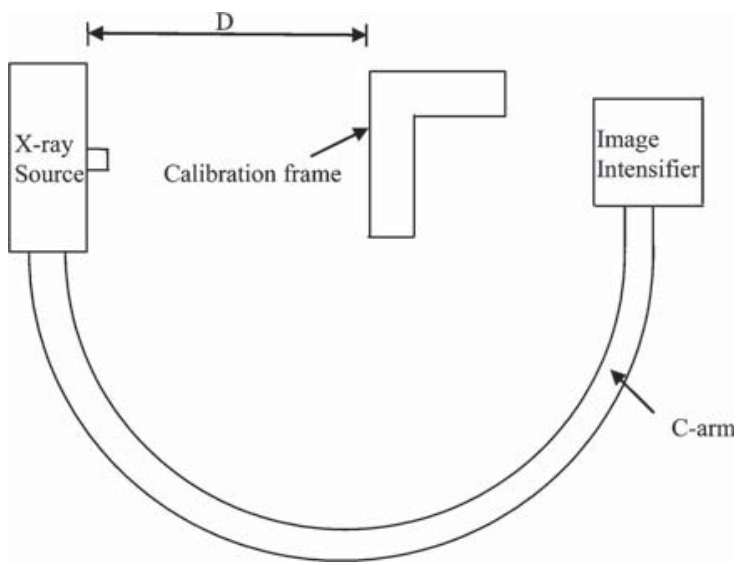

Fig. 13 Positioning of X-ray source relative to calibration frame 
increases. Simulated Gaussian noise was added at various levels between 5 and 15 per cent to each of the variable contrast images using a proprietary image processing package. Subsequent tests showed that good repeatability (less then $0.1^{\circ}$ difference in measurement of the distal hole axis direction) could be obtained for up to 15 per cent of gaussian noise across a wide range of contrast.

\section{DISCUSSION}

Current free-hand techniques employed for distal locking of intramedullary nails use a large number of $\mathrm{X}$-ray images during each step, resulting in excessive irradiation to both the surgeon and patient. Also, the outcome of the procedure is largely dependent upon the surgeon's skills and experience, resulting in variable operating time and, occasionally, misplacement of screws. To reduce surgery time and the surgeon's cumulative radiation exposure, and to improve the accuracy and repeatability of the distal locking procedure, an automated X-ray-based vision system has been developed at Loughborough University. This system minimizes the changes to current surgical procedures and takes into account the physical constraints of the operating theatre, adopting a novel approach based on the use of a calibration frame in conjunction with off-line modelling of the nail.

A potential problem with the Loughborough orthopaedic assistant system (LOAS) is the appearance of one or more of the opaque markers (embedded in the calibration frame plates) within or on the edge of the oval-shaped distal hole projection. An automatic technique has been implemented that removes the effect of such markers in computing the area of the oval and the angle between the major axis of the oval and the nail axis, both of these measurements being necessary for derivation of the drilling trajectory. This technique eliminates the need for repositioning the calibration frame or using a smaller number of opaque markers. Also, the adopted off-line modelling of the nail to generate look-up tables reduces the length of time for the computation of the drilling trajectory and thus has the potential to reduce the overall time required for the surgical procedure. Furthermore, the vision system is user independent as all the steps involved during image analysis are fully automated. A graphical user interface (GUI) has been developed that assists and updates the user throughout the procedure. It has also been shown that the LOAS is very robust in the presence of variable noise and contrast in the X-ray images and in terms of variable magnification, thus making it suitable for clinical use.

The LOAS differs from existing computer-assisted orthopaedic surgery systems, as it eliminates the need for optical tracking equipment which tends to clutter the operating theatre environment and requires care in maintaining the line of sight. Additionally, use of optical tracking equipment makes such systems an expensive method for surgical guidance in distal locking of intramedullary nails. In contrast, the LOAS can be implemented by adopting the simplified approach of using a standard C-arm in conjunction with the calibration frame (shown in Fig. 2). Therefore, the LOAS is more cost effective and more suitable for clinical applications.

During laboratory trials of the LOAS, a mean positional error of $0.81 \mathrm{~mm}$ and a mean angular error of $1.01^{\circ}$ were obtained in planning the drilling trajectory for the insertion of distal locking screws for different orientations of the nail, whereas Phillips et al. [16] at Hull University have reported a mean positional error and a mean angular error within $0.3 \mathrm{~mm}$ and $0.2^{\circ}$ respectively. Although the accuracy achieved by the Hull system is better when compared with the LOAS results, the image analysis protocol and system set-up are user dependent. The system requires the surgeon (or user) manually to select some features on the intraoperative lateral and AP images, and most importantly it requires optical tracking equipment which results in a more involved and time-consuming set-up. The reduced accuracy of the LOAS, which is acceptable for distal locking of intramedullary nails, is a result of a simplified (practical) system that involves the use of a $\Gamma$-shaped calibration frame (shown in Fig. 2) instead of a frame that fits around the leg. That is, the increased errors are mainly due to the adopted extrapolation technique instead of the preferred, but practically difficult to implement, interpolation technique.

Nowadays there is a trend towards applying computer-assisted free-hand navigation systems for different surgical procedures, including distal locking of intramedullary nails. These systems are based on the optical tracking of the C-arm, implant, and surgical tools. However, they do not take into account the bending of the nail during insertion and therefore rely on the realignment of the C-arm to obtain perfect circles of the distal hole during the surgical procedure, which is time consuming and requires a precalibration of the $\mathrm{C}$-arm. Unlike the surgical navigation systems, the LOAS does not require the intraoperatively acquired lateral view image to show perfect circles, and does not need precalibration of 
the C-arm. Also, use of surgical navigation systems requires expertise and additional personnel to set up the navigation system.

In summary, the LOAS has the potential to reduce significantly the X-ray irradiation during distal locking procedures, while the outcome of the procedure will be improved as the system has the potential to reduce the occurrence of screw misplacement, as demonstrated by Fig. 12. Additionally, the LOAS, which is also applicable to other osteosynthesis procedures, such as tibial distal locking and humeral shaft fracture treatment, has the potential to reduce the surgical time and to make the procedure more consistent, irrespective of variations in the skill levels and experience of clinical staff.

\section{REFERENCES}

1 Gray, H. Gray's anatomy: the anatomical basis of clinical practice, 39th edition (Ed. S. Standring), 2005 (Elsevier Churchill Livingstone, Edinburgh).

2 Whatling, G. M. and Nokes, L. D. Literature review of current techniques for the insertion of distal screws into intramedullary locking nails. Injury, 2006, 37(2), 109-119.

3 Krettek, C., Konemann, B., Miclau, T., Kolbli, R., Machreich, T., and Tscherne, H. A mechanical distal aiming device for distal locking in femoral nails. Clinical Orthop. and Relat. Res., 1999, (364), 267-275.

4 Tyropoulos, S. and Garnavos, C. A new distal targeting device for closed interlocking nailing. Injury, 2001, 32(9), 732-735.

5 Goodall, J. D. An image intensifier laser guidance system for the distal locking of an intramedullary nail. Injury, 1991, 22(4), 339.

6 Ohe, T., Nakamura, K., Matsushita, T., Nishiki, M., Watanabe, N., and Matsumoto, K. Stereo fluoroscopy-assisted distal interlocking of intramedullary nails. J. Orthop. Trauma, 1997, 11(4), 300-303.

7 Catamo, L., Rotini, R., Rocca, M., Giardino, R., and Fontanesi, G. Distal centering in locked intramedullary osteosynthesis of the femur: use of a magnet-resistant probe. La Chirurgia Degli Organi Di Movimento, 1998, 83(4), 375-379.

8 Krettek, C., Konemann, B., Miclau, T., Kolbli, R., Machreich, T., Kromm, A., and Tscherne, H. A new mechanical aiming device for the placement of distal interlocking screws in femoral nails. Arch. Orthop. and Trauma Surg., 1998, 117(3), 147-152.

9 Madan, S. and Blakeway, C. Radiation exposure to surgeon and patient in intramedullary nailing of the lower limb. Injury, 2002, 33(8), 723-727.

10 Mehlman, C. T. and DiPasquale, T. G. Radiation exposure to the orthopaedic surgical team during fluoroscopy: 'how far away is far enough?' J. Orthop. Trauma, 1997, 11(6), 392-398.
11 Hafez, M. A., Smith, R. M., Matthews, S. J. Kalap, G., and Sherman, K. P. Radiation exposure to the hands of the surgeons: are we underestimating the risk? Arch. Orthop. and Trauma Surg., 2005, 125, 330-335.

12 Maxon, H. R., Thomas, S. R., Saenger, E. L., Buncher, C. R., and Kereiakes, J. G. Ionizing irradiation and the induction of clinically significant disease in the human thyroid gland. Am. J. Med., 1977, 63(6), 967-978.

13 Hofstetter, R., Slomczykowski, M., Sati, M., and Nolte, L. P. Fluoroscopy as an imaging means for computer assisted surgical navigation. Comput. Aided Surg., 1999, 4(2), 65-76.

14 Slomczykowksi, M. A., Hofstetter, R., Sati, M., Krretek, C., and Nolte, L. P. Novel computer-assisted fluoroscopy system for intraoperative guidance: feasibility study for distal locking of femoral nails. J. Orthop. Trauma, 2001, 15, 122-131.

15 Zhu, Y., Phillips, R., Griffiths, J. G., Viant, W. J., Mohsen, A. M., and Bielby, M. Recovery of distal hole axis in intramedullary nail trajectory planning. Proc. Instn Mech. Engrs, Part H: J. Engineering in Medicine, 2002, 216(H5), 323-332.

16 Malek, S., Phillips, R., Mohsen, A., Viant, W., Bielby, M., and Sherman, K. Computer assisted orthopaedic surgical system for insertion of distal locking screws in intra-medullary nails: a valid and reliable navigation system. Int. J. Med. Robotics and Comput. Assisted Surg., 2006, 1(4), 34-44.

17 Schep, N., Broeders, I., and Van der Werken, C. Computer assisted orthopaedic and trauma surgery: state of the art and future perspectives. Injury, 2003, 34(4), 299-306.

18 Bouazza-Marouf, K., Browbank, I., and Hewit, J. R. Robotic-assisted internal fixation of femoral fractures. Proc. Instn Mech. Engrs, Part H: J. Engineering in Medicine, 1995, 209(H1), 51-58.

19 Bouazza-Marouf, K., Browbank, I., and Hewit, J. R. Robotic-assisted invasive surgery. J. Mechatronics, 1996, 6(4), 381-387.

20 Hochrainer, M. J. Repair of femoral shaft fractures. MSc Thesis, supervised by Dr K. Bouazza-Marouf, Loughborough University, 1997.

21 Browbank, I., Bouazza-Marouf, K., and Schnabler, J. Robotic-assisted internal fixation of hip fractures: a fluoroscopy-based intraoperative registration technique. Proc. Instn Mech. Engrs, Part H: J. Engineering in Medicine, 2000, 214(H2), 165-179.

\section{APPENDIX}

\section{Notation}

$D \quad$ distance between the X-ray source and the calibration frame

$\mathrm{O} \quad$ centre of the distal hole

$\mathrm{P}_{\text {Source }}, \mathrm{P}_{\mathrm{XRII}}$ corresponding points on the Source and the XRII plate for the image 
point representing the centre of the

$\theta$

oval

${ }_{\mathrm{TH}}^{\mathrm{CF}} \mathbf{T}$

homogeneous transformation matrix describing the theodolite

frame of reference relative to the calibration frame coordinate system rotation of the nail about its own axis

angle between the major axis of the oval and the nail axis

angle between the nail axis and the

$\mathrm{X}$-ray imaging axis 